\title{
Síndrome metabólico en pacientes con urolitiasis: una serie actual
}

\section{Metabolic syndrome in patients with urolithiasis: A current case series}

Maricela Martínez-Montoya, ${ }^{1}$ Miguel Ángel Hernández-Farías, ${ }^{2}$ Jorge Jaspersen-Gastelum, ${ }^{3}$ Francisco VirgenGutiérrez, ${ }^{3}$ Alejandro Rosas-Ramírez, ${ }^{3}$ Jorge Jaspersen-Álvarez, ${ }^{4}$ Luis Daniel Carillo-Córdova ${ }^{5}$

\section{Resumen}

ANTECEDENTES: La urolitiasis y el síndrome metabólico son una pandemia mundial con importante repercusión en los sistemas de salud. La incidencia de ambas alteraciones es alta en México, por lo que es importante identificar los factores predisponentes de la urolitiasis y evitar la recurrencia, complicaciones y tratamientos quirúrgicos.

OBJETIVO: Estimar la prevalencia de síndrome metabólico y evaluar las modificaciones en la fisiopatología de la urolitiasis.

MATERIALES Y MÉTODOS: Estudio transversal, no aleatorizado, efectuado en pacientes con diagnóstico de urolitiasis, atendidos en el Hospital General de México Dr. Eduardo Liceaga de marzo a diciembre de 2016. Variables de estudio: medidas antropométricas, toma de presión arterial, concentración de glucosa y perfil de lípidos en ayuno. RESULTADOS: Se registraron 84 pacientes: 32 hombres y 52 mujeres, con edad media de $47.3 \pm 14.1$ años. El $52 \%$ de los casos de urolitiasis correspondió a mujeres. El diagnóstico de síndrome metabólico se estableció en 58 pacientes y de estos $29 \%$ fueron hombres y $71 \%$ mujeres, con una prevalencia-relación de 3:1 (mujer:hombre). CONCLUSIONES: La urolitiasis no debe considerarse una enfermedad aislada, sino un padecimiento multifactorial y enfermedad sistémica. ${ }^{3}$

PALABRAS CLAVE: Síndrome metabólico; obesidad; urolitiasis.

\section{Abstract}

BACKGROUND: Urolithiasis and metabolic syndrome are considered a worldwide pandemic, with important health system repercussions. Mexico has a high incidence of both pathologies. Identifying the predisposing factors of urolithiasis will prevent recurrence, complications, and surgical treatments.

OBJECTIVE: To establish the prevalence of metabolic syndrome and evaluate the modifications in urolithiasis pathophysiology.

MATERIALS AND METHODS: A non-randomized cross-sectional study was conducted on patients seen at the Hospital General de México Dr. Eduardo Liceaga, diagnosed with urolithiasis within the time frame of March to December 2016. Study variables: anthropometric measurements, blood pressure readings, and fasting glucose levels and lipid profiles.

RESULTS: Eighty-four patients, 32 men and 52 women, were enrolled in the study. Their mean age was $47.3 \pm 14.1$ years. A total of $52 \%$ of the cases of urolithiasis corresponded to women. Metabolic syndrome was diagnosed in 58 of the study patients, $29 \%$ of whom were men and $71 \%$ women, representing a woman:man prevalence ratio of 3:1. CONCLUSIONS: Urolithiasis should not be considered an isolated entity, but rather understood in a multifactorial context and as a systemic disease.

KEYWORDS: Metabolic syndrome; Obesity; Urolithiasis.
${ }^{1}$ Licenciada en nutrición, Universidad del Valle de Toluca, Estado de México. ${ }^{2}$ Residente de cuarto año de Urología.

${ }^{3}$ Adscrito al servicio de Urología.

${ }^{4}$ Médico interno de pregrado.

${ }^{5}$ Egresado de Urología.

Hospital General de México Dr. Eduardo Liceaga, Ciudad de México.

Recibido: agosto 2018

Aceptado: octubre 2018

Correspondencia

Miguel Ángel Hernández Farías mdmiguelhernandez@gmail.com

Este artículo debe citarse como Martínez-Montoya M, HernándezFarías MA, Jaspersen-Gastelum J, Virgen-Gutiérrez $F$, Rosas-Ramírez $A$, Jaspersen-Álvarez J, Carillo-Córdova LD. Síndrome metabólico en pacientes con urolitiasis: una serie actual. Rev Mex Urol. 2018 nov-dic;78(6):414-18. DOI: https://doi.org/10.24245/revmexurol.v78i6.2223 


\section{ANTECEDENTES}

Debido a su elevada prevalencia, la urolitiasis y el síndrome metabólico son una pandemia mundial y un problema de salud pública. ${ }^{1-5}$ La incidencia de ambas alteraciones es alta en México, por lo que es importante identificar los factores predisponentes de litiasis urinaria y de esta forma evitar su recurrencia, complicaciones y tratamiento quirúrgico. ${ }^{6}$

El síndrome metabólico es una alteración relacionada con: resistencia a la insulina, obesidad central, hipertensión arterial e hiperlipidemia, ${ }^{1,3,4,7}$ con importante repercusión en el sistema genitourinario. ${ }^{1}$ En la fisiopatología de la urolitiasis intervienen factores metabólicos, genéticos y ambientales. ${ }^{8}$

La fisiopatología de la litiasis urinaria es compleja, incluye volumen urinario bajo, hipercalciuria, hiperuricosuria, hiperoxaluria, hipocitraturia y alteraciones en el $\mathrm{pH}$ urinario que provoca hipersaturación de orina, formación de cristales y, posteriormente, cálculos. ${ }^{2,4,8-10}$ El síndrome metabólico genera disminución del $\mathrm{pH}$ urinario, lo que resulta en depósito de cristales de ácido úrico y formación de cálculos. ${ }^{4}$ Este mecanismo se origina por resistencia a la insulina y en el riñón aumenta la producción de ácidos grasos, que por un mecanismo competitivo con glutamina mitocondrial genera un defecto en la producción de amonio en el túbulo proximal, con subsiguiente disminución del $\mathrm{pH}$ urinario ${ }^{1,5}$ y formación de cálculos de ácido úrico.

La relación entre obesidad, síndrome metabólico y resistencia a la insulina con hiperuricemia e hiperuricosuria está bien establecida. ${ }^{8}$ Se ha estimado que $34 \%$ de los casos con diabetes tipo 2 tiene cálculos de ácido úrico, comparado con $6 \%$ de los pacientes sin diabetes..$^{8,10} \mathrm{El} \mathrm{pH}$ urinario bajo, no relacionado con la dieta y la solución amortiguadora renal defectuosa parecen ser la causa directa de cálculos de ácido úrico en pacientes con síndrome metabólico. ${ }^{1,8,10,11} \mathrm{La}$ solubilidad del ácido úrico es de $15 \mathrm{mg} / \mathrm{dL}$ en un $\mathrm{pH}$ de 5 y de $200 \mathrm{mg} / \mathrm{dL}$ en un $\mathrm{pH}$ de $7 . .^{2,10,12}$ El tratamiento consiste en control del síndrome metabólico, alcalinización de la orina y aumento del consumo de líquidos. ${ }^{10}$

La prevalencia de urolitiasis se duplica en pacientes con cuatro componentes del síndrome metabólico. El síndrome metabólico se conoce como un estado proinflamatorio. Cuando las células renales se dañan o expresan moléculas inflamatorias en su membrana se estimula la adhesión de cristales a su pared. ${ }^{2}$ Diversos estudios señalan que los pacientes con síndrome metabólico tienen 1.8 veces más riesgo de recurrencia de litiasis, además de la formación de múltiples cálculos., ${ }^{43,14}$

La restricción alimenticia y el ejercicio tienen efecto significativo para prevenir y mejorar la sensibilidad a la insulina, además de reducir el riesgo de formación de cálculos de calcio y ácido úrico, aumentar el pH urinario y la excreción de citrato. ${ }^{3,4,12} \mathrm{El}$ análisis del metabolismo es importante en pacientes con urolitiasis, como parte de su tratamiento. ${ }^{12}$

Con base en lo anterior, el objetivo de este estudio fue: estimar la prevalencia de síndrome metabólico y evaluar las modificaciones en la fisiopatología de la urolitiasis.

\section{MATERIALES Y MÉTODOS}

Estudio transversal, no aleatorizado, efectuado en pacientes con diagnóstico de litiasis renal, atendidos en el Hospital General de México Dr. Eduardo Liceaga, entre marzo y diciembre de 2016.

El diagnóstico de síndrome metabólico se estableció con base en tres de cinco criterios de Harmonizing the Metabolic Syndrome: circun- 
ferencia abdominal de la población mexicana (hombres $90 \mathrm{~cm}$ y mujeres $80 \mathrm{~cm}$ ), concentración elevada de triglicéridos (>150 mg/dL), HDL ( $<40 \mathrm{mg} / \mathrm{dL}$ en hombres y $50 \mathrm{mg} / \mathrm{dL}$ en mujeres), tensión arterial mayor de 130/85 mmHg y glucosa en ayuno (> $100 \mathrm{mg} / \mathrm{dL}) .{ }^{11,15} \mathrm{El}$ diagnóstico nutricional se estableció con puntos de corte estandarizados para IMC: $18.5-24.9 \mathrm{~kg} / \mathrm{m}^{2}$ peso normal, $\geq 25-29.9 \mathrm{~kg} / \mathrm{m}^{2}$ sobrepeso, $\geq 30-39.9$ $\mathrm{kg} / \mathrm{m}^{2}$ obesidad y $\geq 40 \mathrm{~kg} / \mathrm{m}^{2}$ obesidad mórbida. Se obtuvieron medidas antropométricas, toma de presión arterial, determinación de glucosa y perfil de lípidos en ayuno.

Para el análisis de los datos se utilizó estadística descriptiva, con medidas de tendencia central y dispersión, mediante el programa SPSS v.22.

\section{RESULTADOS}

Se registraron 108 pacientes, de los que excluyeron 24 por no aceptar participar en el estudio, haber egresado del hospital antes del periodo indicado, perderse en el seguimiento o padecer insuficiencia renal terminal y permanecer en terapia de sustitución. De este modo se incluyeron 84 pacientes: 32 hombres y 52 mujeres, con edad media de $47.3 \pm 14.1$ años. La prevalencia de síndrome metabólico fue de $70 \%$ (Cuadro 1). El diagnóstico de síndrome metabólico se estableció en 58/84 pacientes: 17 hombres y

Cuadro 1. Distribución de pacientes con litiasis según su edad y síndrome metabólico $(n=84)$

\begin{tabular}{|l|c|c|c|c|c|c|}
\hline \multirow{2}{*}{ Variable } & \multicolumn{7}{|c|}{ Edad (años) } \\
\cline { 2 - 7 } & $<\mathbf{2 0}$ & $\mathbf{3 0}$ & $\mathbf{4 0}$ & $\mathbf{5 0}$ & $\mathbf{6 0}$ & $>\mathbf{7 0}$ \\
\hline Hombres & - & 3 & 5 & 2 & 6 & 1 \\
Mujeres & 4 & 6 & 11 & 9 & 8 & 3 \\
\hline Hombres & 3 & 4 & 4 & 3 & 1 & - \\
Mujeres & 4 & 1 & - & 3 & 1 & 2 \\
\hline
\end{tabular}

41 mujeres, con una relación mujer-hombre de

\section{3:1. Figura 1}

La obesidad fue el principal componente del síndrome metabólico: 32 pacientes con sobrepeso, 28 con cierto grado de obesidad y 1 con obesidad mórbida. La obesidad y el sobrepeso fueron tres veces más frecuentes en mujeres que en hombres (Figura 2). La incidencia de urolitiasis también resultó ligeramente mayor en mujeres (52\%), con relación hombre-mujer de 1:1.

\section{DISCUSIÓN}

Hoy día la urolitiasis y el síndrome metabólico se consideran una pandemia mundial y, por lo tanto, un problema de salud pública. ${ }^{1-5}$ La urolitiasis es la tercera enfermedad más frecuente del sistema urinario (5-15\%), seguida de las infecciones de la vía urinaria y las alteraciones prostáticas. ${ }^{8}$ La prevalencia de litiasis urinaria se ha duplicado en las últimas tres décadas en Estados Unidos. En los últimos años se ha modificado la incidencia de litiasis en mujeres, con una relación que varía de $3: 1$ a $2: 1,8,13$ incluso $1: 1$, como lo demuestra esta serie.

La incidencia de síndrome metabólico se reporta en $11.7-42 \%$,-5,14 $^{1-1}$ según la población de estudio y grupos de edad. ${ }^{2,3}$ La prevalencia de urolitiasis se ha incrementado en paralelo con la obesidad

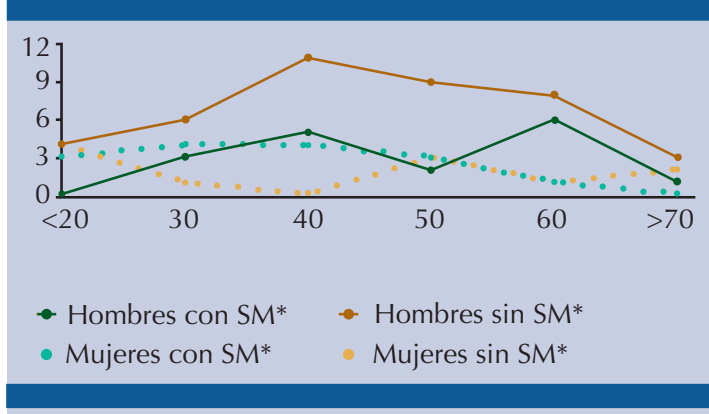

Figura 1. Distribución por edad, sexo y síndrome metabólico.

SM: síndrome metabólico. 


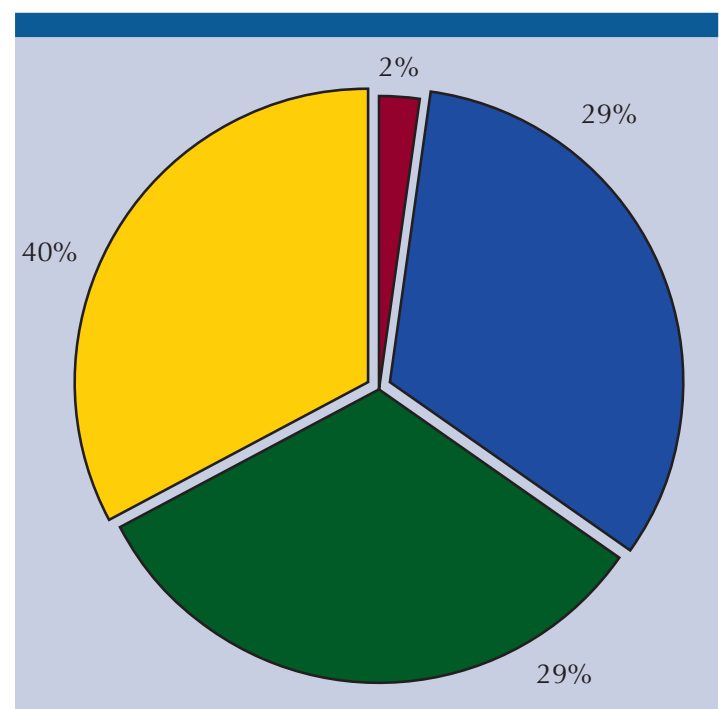

Femenino

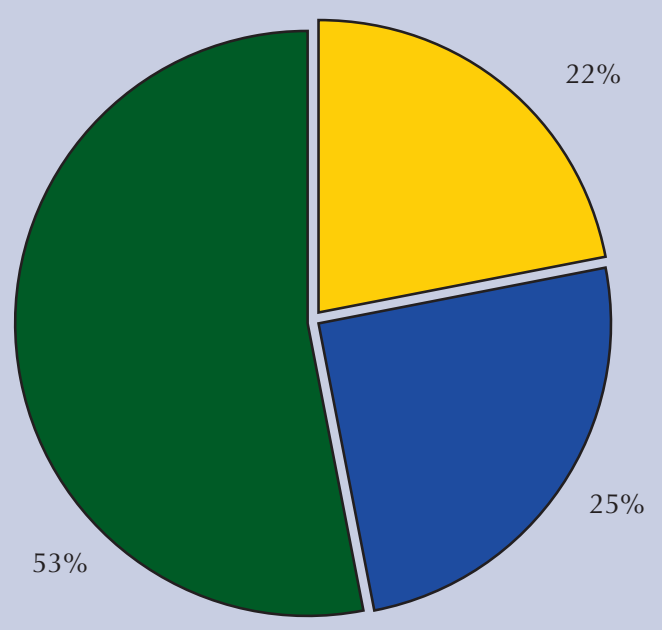

Masculino

Peso normal

$\square$ Obesidad

Sobrepeso

- Obesidad mórbida

Figura 2. Distribución de pacientes según su estado nutricional mediante el cálculo de IMC.

e hipertensión arterial, componentes fundamentales del síndrome metabólico, ${ }^{12,16}$ además de la diabetes tipo 2, cuyo incremento ha sobrepasado las proyecciones internacionales. Este estudio reporta la elevada prevalencia del síndrome metabólico, dato interesante porque ningún paciente conocía este padecimiento.

La obesidad es la manifestación más común del síndrome metabólico. ${ }^{4}$ Un estudio prospectivo de 250,000 pacientes, con seguimiento de 46 años, demostró un riesgo relativo de 1.44 en la incidencia de cálculos renales en hombres de 100 vs $68.2 \mathrm{~kg}(\mathrm{p}=0.002){ }^{8}$ La obesidad es un factor, por sí solo, predisponente para urolitiasis, incluso se ha observado que $75 \%$ de los pacientes con litiasis renal padece sobrepeso, respecto de quienes tienen IMC $\leq 25 \mathrm{~kg} / \mathrm{m}^{2}{ }^{2,7}$ Diversos estudios demuestran que los sujetos con índice de masa corporal $\geq 25 \mathrm{~kg} / \mathrm{m}^{2}$ e hipertensión arterial tienen mayor excreción de ácido úrico, oxalato y fosfato. ${ }^{2,12}$ Como dato interesante, el aumento de IMC se relaciona con baja excreción de citrato. ${ }^{2,13}$ Así mismo, el incremento de IMC se asocia con disminución del $\mathrm{pH}$ urinario. ${ }^{13}$ Por tanto, la obesidad representa un factor de riesgo y recurrencia de urolitiasis. ${ }^{12}$ Se ha sugerido que la mayor tendencia a la obesidad en mujeres confiere un mayor riesgo de urolitiasis. ${ }^{9}$

Debido a la elevada prevalencia de urolitiasis y síndrome metabólico registrada en todo el mundo, es importante modificar los factores de riesgo litogénicos, además de prevenir su recurrencia. Es importante efectuar estudios de orina de 24 horas, con la intención de establecer la causa primaria de la orina litogénica. Además de aumentar el consumo de líquidos, se recomienda disminuir la ingesta de proteínas de origen animal, incluir una dieta baja en sodio y alta en calcio, para de esta forma reducir la tasa de recurrencia de litiasis. Incluso se han descrito beneficios adicionales con el consumo de limón, arándanos, jugo de manzana y citrato relacionados con el aumento del pH urinario y reducción de cálculos renales. En estos pacientes debe complementarse la evaluación médica con el diagnóstico, tratamiento y control del síndrome metabólico. ${ }^{4}$ 


\section{CONCLUSIÓN}

La urolitiasis no debe considerarse una alteración aislada, sino una enfermedad multifactorial, sistémica y crónica implicada con la interacción de diversos factores de riesgo metabólico. ${ }^{6}$ Esta enfermedad debe incluir tratamiento médico e intervención nutricional, para evitar su recurrencia a largo plazo (se estima en 50\% de los casos a 10 años). La identificación de factores predisponentes permite prevenir las complicaciones y tratamiento quirúrgico. El síndrome metabólico debe sospecharse y estudiarse en pacientes con urolitiasis. Este procedimiento permitirá al urólogo establecer un diagnóstico certero y comprensivo, ofrecer el mejor tratamiento, y disminuir la tasa de recurrencia de la urolitiasis. Debido a las modificaciones en las características poblacionales, es necesario efectuar estudios con una muestra mayor de pacientes y segmentarlos de forma estricta para evaluar la repercusión y severidad de cada componente del síndrome metabólico en la urolitiasis.

\section{Financiamiento}

Este trabajo fue financiado por el Hospital General de México con recursos propios de la institución provenientes del sector público sin ánimo de lucro.

\section{REFERENCIAS}

1. Saenz-Medina J, et al. Review of the pathophysiological aspects involved in urological disease associated with metabolic syndrome. Actas Urol Esp 2016;40(5):279-87. DOI: 10.1016/j.acuro.2015.10.001
2. Domingos F, Serra A. Metabolic syndrome: a multifaceted risk factor for kidney stones. Scand J Urol. 2014;48(5):414-9. DOI: 10.3109/21681805.2014.903513

3. Barrera M, Pinilla A, Cortes E, et al. Síndrome metabólico: una mirada interdisciplinaria. Rev Colomb Cardiol 2008;15: 111-126.

4. Wong YV, et al. The association of metabolic syndrome and urolithiasis. Int J Endocrinol. 2015;2015:570674. DOI: $10.1155 / 2015 / 570674$

5. Haymann JP. Metabolic disorders: stones as first clinical manifestation of significant diseases. World J Urol. 2015;33(2):187-92. DOI: 10.1007/s00345-014-1391-5

6. Aragón A, Hernández M. Análisis cristalográfico de 475 cálculos de vías urinarias en el hospital San José, Tec Salud en Monterrey, N.L. Rev Mex Urol 2013;73:130-5.

7. Cho ST, et al. Correlation of metabolic syndrome with urinary stone composition. Int J Urol. 2013;20(2):208-13. DOI: 10.1111/j.1442-2042.2012.03131.x

8. Shadman A, Bastani B. Kidney Calculi: Pathophysiology and as a Systemic Disorder. Iran J Kidney Dis 2017;11(3):180-91.

9. Broadus AE, Thier SO. Metabolic basis of renal-stone disease. N Engl J Med. 1979;300(15):839-45. DOI: 10.1056/ NEJM197904123001507

10. Zerifi R, et al. [Metabolic syndrome: pathophysiology and impact on lithogenesis]. Ann Biol Clin (Paris). 2008;66(1):917. DOI: $10.1684 / a b c .2008 .0187$

11. Lizarzaburu, J. Síndrome metabólico: concepto y aplicación práctica. An Fac med. 2013;74(4):315-20.

12. Wong $Y$, et al. Metabolic Syndrome and Kidney Stone Disease: A Systematic Review of Literature. J Endourol. 2016;30(3):246-53. DOI: 10.1089/end.2015.0567

13. Rendina $D$, et al. Metabolic syndrome and nephrolithiasis: a systematic review and meta-analysis of the scientific evidence. J Nephrol. 2014;27(4):371-6. DOI: 10.1007/ s40620-014-0085-9

14. Lee YC, et al. Impact of metabolic syndrome and its components on kidney stone in aging Taiwanese males. Aging Male 2016;19(3):197-201. DOI: 10.1080/13685538.2016.1174987

15. Rosas J, et al. Epidemiologia, diagnostico, control, prevención y tratamiento del síndrome metabólico en Adultos. ALAD 2010;18(1):25-44.

16. Sasaki $Y$, et al. Weight loss intervention reduces the risk of kidney stone formation in a rat model of metabolic syndrome. Int J Urol. 2015;22(4):404-9. DOI: 10.1111/iju.12691 